Article

\title{
New Type Direction Curves in 3-Dimensional Compact Lie Group
}

\author{
Ali Çakmak (D) \\ Department of Mathematics, Faculty of Science and Arts, Bitlis Eren University, Bitlis 13000, Turkey; \\ alicakmak@yahoo.com; Tel.: +90-530-323-0629
}

Received: 28 February 2019; Accepted: 13 March 2019; Published: 16 March 2019

\begin{abstract}
In this paper, new types of associated curves, which are defined as rectifying-direction, osculating-direction, and normal-direction, in a three-dimensional Lie group $G$ are achieved by using the general definition of the associated curve, and some characterizations for these curves are obtained. Additionally, connections between the new types of associated curves and the curves, such as helices, general helices, Bertrand, and Mannheim, are given.
\end{abstract}

Keywords: Lie group; rectifying-direction curve; osculating-direction curve; normal-direction curve

MSC: 53A04; 22E15

\section{Introduction}

Many authors have made significant contributions to the theory of curves from past to present. Some of these studies indicated that the relationships between the curvatures of the space curves are quite remarkable, and the new special curves are also defined via these relations [1-5]. Helices, one of these special space curves, have been studied by many researchers [6-9]. In addition to special space curves, some of the relationships between the curve pairs are also particularly interesting. The curve pairs are obtained by using the Frenet vectors or curvatures. In this respect, involute-evolute, Bertrand, and Mannheim curves are well-known examples of curve pairs, and many studies have been performed on this topic [10-16].

The Riemannian geometry of a Lie group was studied in [17]. Here, the rich collection of examples that are obtained by providing an arbitrary Lie group $G$ with a Riemannian metric invariant under left translations was given. The semi-Riemannian geometry of a Lie group was examined in [18]. They also obtained the sectional curvature in terms of Lie invariants based on the semisimple case. Furthermore, the curves mentioned above have been handled in Lie group theory by many authors [14,19-22].

In [23], the authors explained the notions of both the principal (binormal)-direction curve and principal (binormal)-donor curve of a Frenet curve in $E^{3}$. They characterized some special curves in $E^{3}$ by using the relationships between the curves.

In this study, within the framework of the definition of associated curves, we introduce new types of direction curves in a three-dimensional Lie group $G$, and we characterize these curves. Finally, we determine the relationships between the new types of direction curves (rectifying-direction, osculating-direction, and normal-direction curve curves) and the curves (Bertrand curve, involute-evolute, rectifying curve, etc.).

\section{Preliminaries}

Suppose that $G$ is a Lie group such that $\langle$,$\rangle is a bi-invariant metric on G$. If the Lie algebra of $G$ is given by $g$, the Lie algebra $g$ is isomorphic to $T_{e} G$, where $e$ is a neutral element of $G$. Since $\langle$,$\rangle is a$ bi-invariant metric on $G$, we get:

$$
\langle X,[Y, Z]\rangle=\langle[X, Y], Z\rangle,
$$


and:

$$
D_{X} Y=\frac{1}{2}[X, Y]
$$

where $X, Y, Z \in g$ and $D$ is the Levi-Civita connection of $G$ [20].

Let us assume that $\alpha: I \subset \mathrm{R} \rightarrow G$ is a curve parameterized by arc-length and $\left\{X_{1}, X_{2}, \ldots, X_{n}\right\}$ is an orthonormal basis of $g$. Now, we consider vector fields $V=\sum_{i=1}^{n} w_{i} X_{i}$ and $Z=\sum_{i=1}^{n} z_{i} X_{i}$ throughout the curve $\alpha$ such that $w_{i}, z_{i}: I \rightarrow \mathrm{R}$ are smooth functions. It is well-known that the Lie bracket of $V$ and $Z$ can be written as:

$$
[V, Z]=\sum_{i=1}^{n} w_{i} z_{i}\left[X_{i}, X_{j}\right]
$$

and $D_{\alpha^{\prime}} V$ is obtained as:

$$
D_{\alpha^{\prime}} V=\dot{V}+\frac{1}{2}[T, V],
$$

where $T=\alpha^{\prime}, \dot{V}=\sum_{i=1}^{n} \dot{w}_{i} X_{i}$ and $D_{\alpha^{\prime}} V$ is the covariant derivative of $V$ throughout the curve $\alpha$ [24].

For the given curve $\alpha$ in $G$, the Frenet formulae are:

$$
D_{T} T=\kappa N, \quad D_{T} N=-\kappa T+\tau B, D_{T} B=-\tau N,
$$

where $\kappa=\|\dot{T}\|$. If $\kappa=\|\dot{T}\| \neq 0, \alpha$ is defined as a Frenet curve [22].

Proposition 1 ([21]). Suppose that the curve $\alpha(s)$ is a curve in Lie group $G$ such that the parameter $s$ is the arc length parameter of $\alpha(s)$, and the Frenet apparatus of $\alpha(s)$ is $(T, N, B, \kappa, \tau)$. Then,

$$
\left\{\begin{array}{l}
{[T, N]=\langle[T, N], B\rangle B=2 \tau_{G} B} \\
{[T, B]=\langle[T, B], N\rangle N=-2 \tau_{G} N .}
\end{array}\right.
$$

Consider that the curve $\alpha(s)$ is a curve in $G$ such that the parameter $s$ is the arc length parameter of $\alpha(s)$. In this case, from (3) and (4), the Frenet formulas are found as follows:

$$
\left(\begin{array}{l}
\frac{d T}{d s} \\
\frac{d N}{d s} \\
\frac{d B}{d s}
\end{array}\right)=\left(\begin{array}{lll}
0 & \kappa & 0 \\
-\kappa & 0 & \tau-\tau_{G} \\
0 & -\left(\tau-\tau_{G}\right) & 0
\end{array}\right)\left(\begin{array}{l}
T \\
N \\
B
\end{array}\right),
$$

where $(T, N, B, \kappa, \tau)$ is the Frenet apparatus of $\alpha$ in $G, \tau_{G}=\frac{1}{2}\langle[T, N], B\rangle[14]$.

Definition 1. Suppose that the curve $\alpha(s)$ is a curve in $G$ such that the parameter sis the arc length parameter of $\alpha(s)$, and the Frenet apparatus of $\alpha(s)$ is $(T, N, B, \kappa, \tau)$. In this case, the harmonic curvature function of $\alpha(s)$ can be given by [21]:

$$
H=\frac{\tau-\tau_{G}}{\kappa}
$$

Theorem 1. Suppose that the curve $\alpha(s)$ is a curve in Lie group $G$ such that $s$ is the arc length parameter of $\alpha(s)$ and the Frenet apparatus of $\alpha(s)$ is $(T, N, B, \kappa, \tau)$. The curve $\alpha$ is a general helix if and only if $\tau=c \kappa+\tau_{G}$, where $c \in \mathbb{R}[20]$.

Thus, the following corollary can be written:

Corollary 1. Suppose that the curve $\alpha$ is a curve in G. Being a general helix in $G$ of $\alpha$ is a necessary and sufficient condition of being $H=$ constant. 
Theorem 2. Suppose that the curve $\alpha$ is an arc length parameterized curve with $(T, N, B, \kappa, \tau)$ in $G$ and $H$ is the harmonic curvature function of $\alpha$. Then, $\alpha$ is a slant helix if and only if the function:

$$
\sigma=\frac{\kappa\left(1+H^{2}\right)^{3 / 2}}{H^{\prime}}
$$

is a constant [21].

\section{Main Results}

In this section, firstly, we mention the existence and definition of a $V$-direction curve. Then, we introduce the concepts of osculating-direction, normal-direction, and rectifying-direction curves in G. We also give some theorems and results characterizing the curves.

Theorem 3. Suppose that $M$ is an n-dimensional Riemannian manifold and $\alpha(t): I \rightarrow U \subset M$ is a curve on a local chart $U$ of $M$. Then, for a continuous vector field $V=V(t)$ on $U \subset M$ along $\alpha$, there exists a curve $\gamma(t): I \subset \mathrm{R} \rightarrow M$ on the neighborhood $U$ such that $\gamma^{\prime}(t)=V(t)$ [25].

Remark 1. For any point $p=\alpha\left(t_{0}\right)$, there is $J \subset I$ including $t_{0}$ and the curve $\gamma(t)$ on neighborhood $U$ satisfying $\gamma\left(t_{0}\right)=p$ and $\frac{d}{d t} \gamma(t)=\left.V(t)\right|_{U}$ for all $t \in J$ [25].

Definition 2. Suppose that $\alpha$ is a curve in $M$ and $p$ is any point on $\alpha$. In this case, the above-mentioned curve $\gamma$ is named as the $V$-direction curve passing the point $p$ of $\alpha$, and also, $\alpha$ is named as the $V$-donor curve passing the point $p$ of $\gamma$.

Since a Lie group is a differentiable manifold in terms of structural properties, Definition 2 can be handled in $G$. Then, we take into account a Frenet curve $\alpha$ in $G$ with the Frenet frame $\{T, N, B\}$ and a unit vector field $V$ given by:

$$
V(s)=u(s) T(s)+v(s) N(s)+w(s) B(s),
$$

where:

$$
u^{2}(s)+v^{2}(s)+w^{2}(s)=1 .
$$

$s$ is the arc length parameter of $\alpha$, and $u, v$ and $w$ are arbitrary differentiable functions [25].

\subsection{Osculating-Direction Curves}

Definition 3. Suppose that $\alpha$ is a Frenet curve in $G$ and $W$ is a unit vector field lying on the osculating plane of $\alpha$ and defined by:

$$
W(s)=u(s) T(s)+v(s) N(s), \quad u(s) \neq 0, v(s) \neq 0
$$

such that the vectors $W^{\prime}(s)$ and $B(s)$ are linearly dependent. Let $\gamma: I \rightarrow G$ be an integral curve of $W(s)$. In this case, $\gamma$ is defined as an osculating-direction curve of $\alpha$, and the curve $\alpha$ is defined as an osculating-donor curve in $G$.

Since $W(s)$ is a unit vector field and $\gamma: I \rightarrow G$ is an integral curve of $W(s)$, without loss of generality, we can take $s$ as the arc length parameter of $\gamma$, and we can give the following characterizations in view of these facts.

Theorem 4. Suppose that $\alpha$ is a Frenet curve in $G$ and the curve $\gamma$ is an integral curve of $W=u(s) T(s)+$ $v(s) N(s)$. Then, $\gamma$ is an osculating-direction curve of $\alpha$ if and only if:

$$
u(s)=\sin \left(\int \kappa d s\right) \neq 0, \quad v(s)=\cos \left(\int \kappa d s\right) \neq 0 .
$$


Proof. Since $\gamma$ is the osculating-direction curve of $\alpha$, from Definition 3, we have:

$$
W(s)=u(s) T(s)+v(s) N(s),
$$

and:

$$
u^{2}(s)+v^{2}(s)=1 .
$$

By differentiating (11) according to $s$, we get:

$$
\frac{d}{d s} W(s)=u^{\prime}(s) T(s)+v^{\prime}(s) N(s)+u(s)\left(D_{T} T-\frac{1}{2}[T, T]\right)+v(s)\left(D_{T} N-\frac{1}{2}[T, N]\right) .
$$

Furthermore, from the Frenet formulas and (13), we obtain:

$$
W^{\prime}(s)=\left(u^{\prime}-v \kappa\right) T+\left(v^{\prime}+u \kappa\right) N+v \kappa H B,
$$

where $H$ is the harmonic curvature function.

Since $W^{\prime} \notin S p\{T, N\}$, we have that $W^{\prime}$ and $B$ are linearly dependent. From (14), we can write:

$$
\left\{\begin{array}{l}
v \kappa H \neq 0, \\
u^{\prime}-v \kappa=0, \\
v^{\prime}+u \kappa=0 .
\end{array}\right.
$$

The solutions of last two differential equations are:

$$
u(s)=\sin \left(\int \kappa d s\right) \neq 0, \quad v(s)=\cos \left(\int \kappa d s\right) \neq 0
$$

respectively, which completes the proof.

Theorem 5. Suppose that $\alpha: I \rightarrow G$ is a Frenet curve in $G$. If the curve $\gamma$ is the osculating-direction curve of $\alpha$, then $\gamma$ is a Mannheim curve of $\alpha$.

Proof. Since $\gamma$ is an integral curve of $W$, we have $\gamma^{\prime}=W$. Let $\{\bar{T}, \bar{N}, \bar{B}\}$ be the Frenet frame of $\gamma$. Differentiating $\gamma^{\prime}=W$ according to $s$, we get:

$$
\begin{aligned}
\frac{d}{d s} W(s) & =\bar{T}^{\prime}=D_{\bar{T}} \bar{T}-\frac{1}{2}[\bar{T}, \bar{T}], \\
W^{\prime} & =\bar{T}^{\prime}=\bar{\kappa} \bar{N} .
\end{aligned}
$$

Moreover, we know that $W^{\prime}$ and $B$ are linearly dependent. From (17), $\bar{N}$ and $B$ are linearly dependent, i.e., $\gamma$ is a Mannheim curve of $\alpha$.

Theorem 6. Suppose that $\alpha: I \rightarrow G$ is a Frenet curve in $G$. If the curve $\gamma$ is the osculating-direction curve of $\alpha$, the curvature $\bar{\kappa}$ and the torsion $\bar{\tau}$ of the curve $\gamma$ can be written, respectively,

$$
\bar{\kappa}=\kappa H \cos \left(\int \kappa d s\right), \quad \bar{\tau}-\bar{\tau}_{g}=-\kappa H \sin \left(\int \kappa d s\right) .
$$

Proof. From (14) and (17), we have:

$$
\bar{\kappa} \bar{N}=v \kappa H B .
$$

By considering (10) and (19), we obtain:

$$
\bar{\kappa} \bar{N}=\kappa H \cos \left(\int \kappa d s\right) B,
$$


which gives us:

$$
\bar{\kappa}=\kappa H \cos \left(\int \kappa d s\right) .
$$

Besides, from (20) and (21), we can write:

$$
\bar{N}=B
$$

Then, we have:

$$
\bar{B}=\bar{T} \times \bar{N}=W \times B=\cos \left(\int \kappa d s\right) T-\sin \left(\int \kappa d s\right) N .
$$

Differentiating (23) according to $s$, we have:

$$
\frac{d}{d s} \bar{B}=D_{\bar{T}} \bar{B}-\frac{1}{2}[\bar{T}, \bar{B}]=\kappa H \sin \left(\int \kappa d s\right) B,
$$

where $H$ is the harmonic curvature function. Since $\frac{d}{d s} \bar{B}=-\left(\bar{\tau}-\bar{\tau}_{g}\right) \bar{N}$, Equation (24) gives us:

$$
\bar{\tau}-\bar{\tau}_{g}=-\kappa H \sin \left(\int \kappa d s\right)
$$

Hence, the proof is completed.

Theorem 7. Suppose that $\alpha: I \rightarrow G$ is a Frenet curve in $G$. If the curve $\gamma$ is the osculating-direction curve of $\alpha$, the relations between the harmonic curvature functions are given as follows:

$$
H=-\frac{\bar{H}^{\prime}}{\bar{\kappa}\left(1+\bar{H}^{2}\right)^{3 / 2}}
$$

where $H$ and $\bar{H}$ are, respectively, harmonic curvature functions of $\alpha$ and $\gamma$.

Proof. Using (21), (25) and the equation $\bar{H}=\frac{\bar{\tau}-\bar{\tau}_{g}}{\overline{\bar{\kappa}}}$, we easily get:

$$
\kappa H=\bar{\kappa} \sqrt{\left(1+\bar{H}^{2}\right)} .
$$

Substituting (27) into (21) and (25), it follows:

$$
\begin{gathered}
\sin \left(\int \kappa d s\right)=-\frac{\bar{\tau}-\bar{\tau}_{g}}{\bar{\kappa} \sqrt{\left(1+\bar{H}^{2}\right)}}=-\frac{\bar{H}}{\sqrt{1+\bar{H}^{2}}}, \\
\cos \left(\int \kappa d s\right)=\frac{\bar{\kappa}}{\bar{\kappa} \sqrt{\left(1+\bar{H}^{2}\right)}}=\frac{1}{\sqrt{1+\bar{H}^{2}}},
\end{gathered}
$$

respectively. From (28):

$$
\kappa \cos \left(\int \kappa d s\right)=-\frac{\bar{H}^{\prime} \cdot \sqrt{1+\bar{H}^{2}}-\bar{H} \cdot \frac{\bar{H} \cdot \bar{H}^{\prime}}{\sqrt{1+\bar{H}^{2}}}}{1+\bar{H}^{2}} .
$$

Substituting (29) into (30), we obtain:

$$
\kappa=-\frac{\bar{H}^{\prime}}{1+\bar{H}^{2}}
$$


and from (27) and (31), it follows:

$$
H=-\frac{\bar{H}^{\prime}}{\bar{\kappa}\left(1+\bar{H}^{2}\right)^{3 / 2}}
$$

which finishes the proof.

Thus, Theorem 7 immediately gives the following conclusion.

Corollary 2. Suppose that $\alpha: I \rightarrow G$ is a Frenet curve in $G$ and the curve $\gamma$ is an osculating-direction curve of $\alpha$. Then:

(i) $\alpha$ is a general helix in $G$.

(ii) $\alpha$ is an osculating-donor curve of a slant helix.

(iii) $\gamma$ is a slant helix.

\subsection{Normal-Direction Curves}

Definition 4. Suppose that $\alpha$ is a Frenet curve in $G$ and $X$ is a unit vector field lying on the normal plane of $\alpha$ and defined by:

$$
X(s)=\eta(s) N(s)+\vartheta(s) B(s), \eta(s) \neq 0, \vartheta(s) \neq 0
$$

such that the vectors $X^{\prime}(s)$ and $T(s)$ are linearly dependent. Let $\gamma: I \rightarrow G$ be an integral curve of $X(s)$. In this case, $\gamma$ is defined as a normal-direction curve of $\alpha$, and the curve $\alpha$ is defined as a normal-donor curve in $G$.

Theorem 8. Suppose that $\alpha$ is a Frenet curve in $G$ and the curve $\gamma$ is an integral curve of $X=\eta(s) N(s)+$ $\vartheta(s) B(s)$. Then, $\gamma$ is a normal-direction curve of $\alpha$ if and only if:

$$
\eta(s)=\sin \left(\int \kappa H d s\right) \neq 0, \quad \vartheta(s)=\cos \left(\int \kappa H d s\right) \neq 0 .
$$

Proof. Since $\gamma$ is a normal-direction curve of $\alpha$, we obtain:

$$
X(s)=\eta(s) N(s)+\vartheta(s) B(s),
$$

and:

$$
\eta^{2}(s)+\vartheta^{2}(s)=1 .
$$

Differentiating (34) with respect to $s$ :

$$
\begin{aligned}
\frac{d}{d s} X(s)= & \eta^{\prime}(s) N(s)+\vartheta^{\prime}(s) B(s) \\
& +\eta(s)\left(D_{T} N-\frac{1}{2}[T, N]\right)+\vartheta(s)\left(D_{T} B-\frac{1}{2}[T, B]\right)
\end{aligned}
$$

and by using the Frenet formulas, we get:

$$
X^{\prime}(s)=-\eta \kappa T+\left(\eta^{\prime}-\vartheta \kappa H\right) N+\left(\vartheta^{\prime}+\eta \kappa H\right) B .
$$

Since $X^{\prime} \notin S p\{N, B\}$, we have that $X^{\prime}$ and $T$ are linearly dependent. Then, from (37), we can write:

$$
\left\{\begin{array}{l}
-\eta \kappa \neq 0, \\
\eta^{\prime}-\vartheta \kappa H=0, \\
\vartheta^{\prime}+\eta \kappa H=0 .
\end{array}\right.
$$


The solutions of last two differential equations are:

$$
\eta(s)=\sin \left(\int \kappa H d s\right) \neq 0, \quad \vartheta(s)=\cos \left(\int \kappa H d s\right) \neq 0,
$$

respectively, which completes the proof.

Theorem 9. Suppose that $\alpha: I \rightarrow G$ is a Frenet curve in $G$. If $\gamma$ is the normal-direction curve of $\alpha$, the curve $\gamma$ is an evolute of $\alpha$.

Proof. Since $\gamma$ is an integral curve of $X$, we have $\gamma^{\prime}=X$. Denote the Frenet frame of $\gamma$ by $\{\bar{T}, \bar{N}, \bar{B}\}$. Differentiating $\gamma^{\prime}=X$ according to $s$, we have:

$$
\begin{aligned}
\frac{d}{d s} X(s) & =\bar{T}^{\prime}=D_{\bar{T}} \bar{T}-\frac{1}{2}[\bar{T}, \bar{T}] \\
X^{\prime} & =\bar{T}^{\prime}=\bar{\kappa} \bar{N} .
\end{aligned}
$$

Furthermore, we know that $X^{\prime}$ and $T$ are linearly dependent. From (40), we get that $\bar{N}$ and $T$ are linearly dependent, i.e., $\gamma$ is a space evolute of $\alpha$.

Theorem 10. Suppose that $\alpha: I \rightarrow G$ is a Frenet curve in $G$. If $\gamma$ is the normal direction curve of $\alpha$, the curvature $\bar{\kappa}$ and the torsion $\bar{\tau}$ of the curve $\gamma$ can be written, respectively,

$$
\bar{\kappa}=\kappa \sin \left(\int \kappa H d s\right), \quad \bar{\tau}-\bar{\tau}_{g}=\kappa \cos \left(\int \kappa H d s\right) .
$$

Proof. From (37), (38), and (40), we have:

$$
\bar{\kappa} \bar{N}=-\eta \kappa T .
$$

By considering (42) and (33), we obtain:

$$
\bar{\kappa} \bar{N}=-\kappa \sin \left(\int \kappa H d s\right) T,
$$

which gives us:

$$
\bar{\kappa}=\kappa \sin \left(\int \kappa H d s\right) .
$$

Moreover, from (43) and (44), we can write:

$$
\bar{N}=T .
$$

Then, we have:

$$
\bar{B}=\bar{T} \times \bar{N}=\cos \left(\int \kappa H d s\right) N-\sin \left(\int \kappa H d s\right) B .
$$

By differentiating (46) according to $s$, we have:

$$
\frac{d}{d s} \bar{B}=D_{\bar{N}} \bar{B}-\frac{1}{2}[\bar{N}, \bar{B}]=-\kappa \cos \left(\int \kappa H d s\right) T .
$$

Since $\frac{d}{d s} \bar{B}=-\left(\bar{\tau}-\bar{\tau}_{g}\right) \bar{N}$, Equation (47) gives us:

$$
\bar{\tau}-\bar{\tau}_{g}=\kappa \cos \left(\int \kappa H d s\right),
$$


which finishes the proof.

Theorem 11. Suppose that $\alpha: I \rightarrow G$ is a Frenet curve in $G$. If $\gamma$ is the normal-direction curve of $\alpha$, the relations between the harmonic curvature functions are given as follows:

$$
H=-\frac{\bar{H}^{\prime}}{\bar{\kappa}\left(1+\bar{H}^{2}\right)^{3 / 2}}
$$

where $H$ and $\bar{H}$ are, respectively. harmonic curvature functions of $\alpha$ and $\gamma$.

Proof. From Equations (44) and (48), and $\bar{H}=\frac{\bar{\tau}-\bar{\tau}_{g}}{\bar{\kappa}}$, we easily get:

$$
\kappa=\sqrt{\bar{\kappa}^{2}\left(1+\bar{H}^{2}\right)} .
$$

Substituting (50) into (44) and (48), it follows:

$$
\begin{aligned}
& \sin \left(\int \kappa H d s\right)=\frac{\kappa}{\sqrt{\bar{\kappa}^{2}\left(1+\bar{H}^{2}\right)}}=\frac{1}{\sqrt{1+\bar{H}^{2}}}, \\
& \cos \left(\int \kappa H d s\right)=\frac{\bar{\kappa} \bar{H}}{\sqrt{\bar{\kappa}^{2}\left(1+\bar{H}^{2}\right)}}=\frac{\bar{H}}{\sqrt{1+\bar{H}^{2}}},
\end{aligned}
$$

respectively. Differentiating (51) with respect to $s$, we get:

$$
\kappa H \cos \left(\int \kappa H d s\right)=-\frac{\bar{H} \cdot \bar{H}^{\prime}}{\left(1+\bar{H}^{2}\right)^{3 / 2}} .
$$

Substituting (52) into (53), we obtain:

$$
\kappa H=-\frac{\bar{H}^{\prime}}{1+\bar{H}^{2}}
$$

and from (50) and (54), it follows:

$$
H=-\frac{\bar{H}^{\prime}}{\bar{\kappa}\left(1+\bar{H}^{2}\right)^{3 / 2},}
$$

which finishes the proof.

Thus, the above theorem gives the following result.

Corollary 3. Suppose that $\alpha: I \rightarrow G$ is a Frenet curve in $G$ and $\gamma$ is a normal-direction curve of $\alpha$. Then:

(i) $\alpha$ is a general helix in $G$.

(ii) $\alpha$ is a normal-donor curve of a slant helix

(iii) $\gamma$ is a slant helix.

\subsection{Rectifying-Direction Curves}

Definition 5. Suppose that $\alpha$ is a Frenet curve in $G$ and $Y$ is a unit vector field lying on the rectifying plane of $\alpha$ and defined by:

$$
Y(s)=\varsigma(s) T(s)+\omega(s) B(s), \varsigma(s) \neq 0, \omega(s) \neq 0
$$

such that the vectors $Y^{\prime}(s)$ and $N(s)$ are linearly dependent. Let $\gamma: I \rightarrow G$ be an integral curve of $Y(s)$. In this case, $\gamma$ is defined as a rectifying-direction curve of $\alpha$, and the curve $\alpha$ is defined as a rectifying-donor curve in $G$. 
Theorem 12. Suppose that $\alpha$ is a Frenet curve in $G$ and $\gamma$ is an integral curve of $Y(s)=\varsigma(s) T(s)+\omega(s) B(s)$. Then, $\gamma$ is a rectifying-direction curve of $\alpha$ if and only if:

$$
Y(s)=c_{1} T(s)+c_{2} B(s),
$$

where $c_{1}, c_{2}$ are non-zero constants.

Proof. Since $\gamma$ is a rectifying-direction curve of $\alpha$, we have:

$$
Y(s)=\varsigma(s) T(s)+\omega(s) B(s),
$$

and:

$$
s^{2}(s)+\omega^{2}(s)=1 .
$$

By differentiating (57) according to $s$, it follows:

$$
\begin{aligned}
\frac{d}{d s} Y(s)= & \varsigma^{\prime}(s) T(s)+\omega^{\prime}(s) B(s) \\
& +\varsigma(s)\left(D_{T} T-\frac{1}{2}[T, T]\right)+\omega(s)\left(D_{T} B-\frac{1}{2}[T, B]\right)
\end{aligned}
$$

and by using the Frenet formulas, we get:

$$
\Upsilon^{\prime}(s)=\varsigma^{\prime} T+(\varsigma \kappa-\omega \kappa H) N+\omega^{\prime} B .
$$

Here, $Y^{\prime}$ and $N$ are linearly dependent. From (60), we can write:

$$
\left\{\begin{array}{l}
\varsigma^{\prime}=0, \\
\varsigma^{\mathcal{K}}-\boldsymbol{\omega} \kappa H \neq 0, \\
\boldsymbol{\omega}^{\prime}=0 .
\end{array}\right.
$$

The solutions of the first and third differential equations are:

$$
\varsigma(s)=c_{1}=\text { constant }, \quad \omega(s)=c_{2}=\text { constant },
$$

respectively, which completes the proof.

Since (56) is a unit vector, we can write:

$$
Y(s)=\cos \theta T(s)+\sin \theta B(s),
$$

where $\theta$ is the angle between unit vectors $Y$ and $T$. Hence, we have the following corollary.

Corollary 4. The angle $\theta$ between rectifying-direction curve $\gamma$ and its rectifying-donor curve $\alpha$ is constant.

Theorem 13. Suppose that $\alpha: I \rightarrow G$ is a Frenet curve in $G$. If $\gamma$ is the rectifying-direction curve of $\alpha$, the curve $\gamma$ is a Bertrand curve of $\alpha$.

Proof. Since $\gamma$ is an integral curve of $Y$, we have $\gamma^{\prime}=Y$. Let $\{\bar{T}, \bar{N}, \bar{B}\}$ be the Frenet frame of $\gamma$. By differentiating $\gamma^{\prime}=Y$ according to $s$, we get:

$$
\begin{gathered}
\frac{d}{d s} Y(s)=\bar{T}^{\prime}=D_{\bar{T}} \bar{T}-\frac{1}{2}[\bar{T}, \bar{T}] \\
Y^{\prime}=\bar{T}^{\prime}=\bar{\kappa} \bar{N},
\end{gathered}
$$


where $\bar{\kappa}$ is the curvature of $\gamma$. Furthermore, we know that $Y^{\prime}$ and $N$ are linearly dependent. This means that $\bar{N}$ and $N$ are linearly dependent, i.e., $\gamma$ is a Bertrand curve of $\alpha$.

Theorem 14. Suppose that $\alpha: I \rightarrow G$ is a Frenet curve. If $\gamma$ is the rectifying-direction curve of $\alpha$, the curvature $\bar{\kappa}$ and the torsion $\bar{\tau}$ of the curve $\gamma$ can be written, respectively,

$$
\bar{\kappa}=\kappa \cos \theta-\kappa H \sin \theta, \quad \bar{\tau}-\bar{\tau}_{g}=\kappa \sin \theta-\kappa H \cos \theta .
$$

Proof. From Theorem 13, we have:

$$
Y^{\prime}=\bar{\kappa} \bar{N} .
$$

By differentiating $Y(s)=\cos \theta T(s)+\sin \theta B(s)$ according to $s$, we obtain:

$$
Y^{\prime}(s)=(\kappa \cos \theta-\kappa H \sin \theta) N .
$$

Thus, from (67) and (68), we get:

$$
\bar{\kappa}=\kappa \cos \theta-\kappa H \sin \theta .
$$

Since:

$$
\bar{N}=N,
$$

from (63), it follows:

$$
\bar{B}=\bar{T} \times \bar{N}=-\sin \theta T+\cos \theta B .
$$

By differentiating (71) according to $s$ and using the Frenet formulas, we find:

$$
\bar{B}^{\prime}=(\kappa H \cos \theta-\kappa \sin \theta) N .
$$

Since $\frac{d}{d s} \bar{B}=-\left(\bar{\tau}-\bar{\tau}_{g}\right) \bar{N}$, we have:

$$
\bar{\tau}-\bar{\tau}_{g}=\kappa \sin \theta-\kappa H \cos \theta,
$$

where $H$ is the harmonic curvature function. Hence, the proof is finished.

Corollary 5. Suppose that $\gamma$ is the rectifying-direction curve of the curve $\alpha$. In this case, the relationship between Frenet vectors can be written as:

$$
Y=\bar{T}=\cos \theta T+\sin \theta B, \quad \bar{N}=N, \quad \bar{B}=-\sin \theta T+\cos \theta B
$$

or:

$$
T=\cos \theta \bar{T}-\sin \theta \bar{B}, \quad N=\bar{N}, \quad B=\sin \theta \bar{T}+\cos \theta \bar{B} .
$$

Corollary 6. Suppose that $\gamma$ is the rectifying-direction curve of the curve $\alpha$ with $\bar{\kappa}$ and $\bar{\tau}$. Then,

$$
\kappa=\bar{\kappa} \cos \theta+\left(\bar{\tau}-\bar{\tau}_{g}\right) \sin \theta, \quad \tau-\tau_{g}=-\bar{\kappa} \sin \theta+\left(\bar{\tau}-\bar{\tau}_{g}\right) \cos \theta,
$$

where $\kappa$ and $\tau$ are the curvature and the torsion of $\alpha$, respectively.

Theorem 15. The rectifying-donor curve $\alpha$ of the curve $\gamma$ is not a general helix.

Proof. From the second system of Equation (61), we have that the function $\frac{\tau-\tau_{g}}{\kappa}(s)$ is not a constant. 
Now, we investigate the condition of the existence for rectifying-direction curve $\gamma$ to be a general helix. If we assume that rectifying-direction curve $\gamma$ is a general helix, from Theorem 14,

$$
\bar{H}=\frac{\bar{\tau}-\bar{\tau}_{g}}{\bar{\kappa}}(s)=\frac{c \cos \theta-\sin \theta}{c \sin \theta-\cos \theta}
$$

is a constant. Then, from (77) and Corollary 4, it follows that the function:

$$
H=\frac{\tau-\tau_{g}}{\kappa}(s)=\frac{\cos \theta-\sin \theta \bar{H}}{-\sin \theta-\cos \theta \bar{H}}
$$

is a constant, i.e., $\alpha$ is a general helix. By considering Theorem 14, we obtain a contradiction.

Thus, Theorem 15 gives the following conclusion.

Corollary 7. Suppose that $\alpha: I \rightarrow G$ is a Frenet curve in $G$ and $\gamma$ is a rectifying-direction curve of $\alpha$. Then,

(i) $\alpha$ is not a general helix in $G$.

(ii) $\alpha$ is not a rectifying-donor curve of a general helix.

(iii) A rectifying-direction curve $\gamma$ of $\alpha$ is not a general helix.

\section{Conclusions}

In this study, the new types of direction curves in a three-dimensional Lie group $G$ are introduced. The curvature and torsion functions are calculated via the Frenet frame expressed for these curves. By using these curvatures, under what circumstances and conditions new types of direction curves occur, such as helix, slant helix, Bertrand, Mannheim, and involute-evolute curves, are discussed.

Funding: This research received no external funding.

Conflicts of Interest: The authors declare no conflict of interest.

\section{References}

1. Barros, M. General helices and a theorem of Lancret. Proc. Am. Math. Soc. 1997, 125, 1503-1509. [CrossRef]

2. Hayden, H.A. On a generalized helix in a Riemannian n-space. Proc. Lond. Math. Soc. 1931, 2, $337-345$. [CrossRef]

3. Izumiya, S.; Takeuchi, N. New special curves and developable surfaces. Turk. J. Math. 2004, 28, $153-163$.

4. Struik, D.J. Lectures on Classical Differential Geometry, 2nd ed.; Courier Corporation: New York, NY, USA, 2012.

5. Wong, Y.C. On the generalized helices of Hayden and Syptak in an N-space. Proc. Camb. Philos. Soc. 1941, 37, 229-243. [CrossRef]

6. Beltran, J.V.; Monterde J. A characterization of quintic helices. J. Comput. Appl. Math. 2007, 206, $116-121$. [CrossRef]

7. Da Fonseca, A.F.; Malta, C.P. Lancret helices. arXiv 2005, arXiv:physics/0507105v1.

8. Farouki, R.T.; Han, C.Y.; Manni, C.; Sestini, A. Characterization and construction of helical polynomial space curves. J. Comput. Appl. Math. 2004, 162, 365-392. [CrossRef]

9. Puig-Pey, J.; Gálvez, A.; Iglesias, A. Helical curves on surfaces for computer-aided geometric design and manufacturing. In International Conference on Computational Science and Its Applications-ICCSA 2004; Springer: Berlin/Heidelberg, Germany, 2004; pp. 771-778.

10. Burke, J.F. Bertrand curves associated with a pair of curves. Math. Mag. 1960, 34, 60-62. [CrossRef]

11. Izumiya, S.; Takeuchi, N. Generic properties of helices and Bertrand curves. J. Geom. 2002, 74, 97-109. [CrossRef]

12. Kahraman, T.; Önder, M.; Kazaz, M.; Uğurlu, H.H. Some Characterizations of Mannheim Partner Curves in Minkowski 3-space. Proc. Est. Acad. Sci. 2011, 60, 210-220. [CrossRef]

13. Liu, H.; Wang, F. Mannheim partner curves in 3-space. J. Geom. 2008, 88, 120-126. [CrossRef]

14. Okuyucu, O.Z.; Yıldız, Ö.G.; Tosun, M. Spinor Frenet equations in three dimensional Lie groups. arXiv 2012, arXiv:1212.6560v1. 
15. Wang, F.; Liu, H. Mannheim partner curves in 3-Euclidean space. Math. Pract. Theory 2007, 37, 141-143.

16. Whittemore, J.K. Bertrand curves and helices. Duke Math. J. 1940, 6, 235-245. [CrossRef]

17. Milnor, J. Curvatures of Left Invariant Metrics on Lie Groups. Adv. Math. 1976, 21, 293-329. [CrossRef]

18. Çöken, A.C.; Çiftçi, Ü. A note on the geometry of Lie groups. Nonlinear Anal. TMA 2008, 68, $2013-2016$. [CrossRef]

19. Bozkurt, Z.; Gök, I.; Okuyucu, O.Z.; Ekmekçi, F.N. Characterizations of rectifying, normal and osculating curves in three dimensional compact Lie groups. Life Sci. J. 2013, 10, 819-823.

20. Çiftçi, Ü. A generalization of Lancret's theorem. J. Geom. Phys. 2009, 59, 1597-1603. [CrossRef]

21. Okuyucu, O.Z.; Gök, İ.; Yaylı, Y.; Ekmekçi, F.N. Slant helices in three dimensional Lie groups. Appl. Math. Comput. 2013, 221, 672-683.

22. Okuyucu, O.Z.; Gök, İ.; Yaylı, Y.; Ekmekçi, F.N. Bertrand curves in three dimensional Lie groups. arXiv 2012, arXiv:1211.6424v1.

23. Choi, J.H.; Kim, Y.H. Associated curves of a Frenet curve and their applications. Appl. Math. Comput. 2012, 218, 9116-9124. [CrossRef]

24. Crouch, P.; Silva, L.F. The dynamic interpolation problem: On Riemannian manifolds, Lie groups, and symmetric spaces. J. Dyn. Control Syst. 1995, 1, 177-202. [CrossRef]

25. Kızıltug, S.; Önder, M. Associated Curves of Frenet Curves in Three Dimensional Compact Lie Group. Miskolc Math. Notes 2015, 16, 953-964. [CrossRef]

(C) 2019 by the author. Licensee MDPI, Basel, Switzerland. This article is an open access article distributed under the terms and conditions of the Creative Commons Attribution (CC BY) license (http:/ / creativecommons.org/licenses/by/4.0/). 